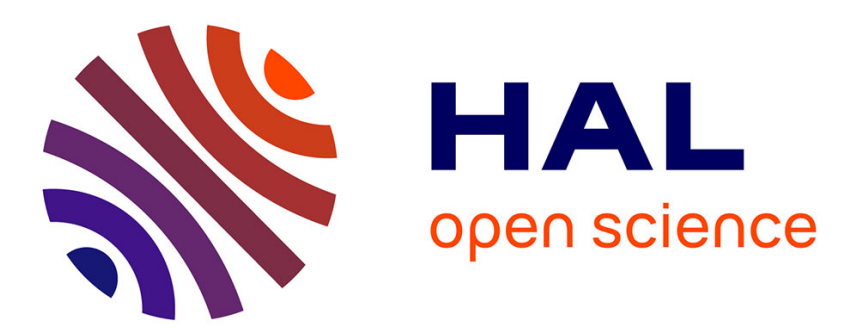

\title{
Estimation of the Lyman- $\alpha$ signal of the EFILE diagnostic under static or radiofrequency electric field in vacuum
}

Carlo Poggi, Théo Guillaume, Fabrice Doveil, Laurence Chérigier-Kovacic

\section{- To cite this version:}

Carlo Poggi, Théo Guillaume, Fabrice Doveil, Laurence Chérigier-Kovacic. Estimation of the Lyman- $\alpha$ signal of the EFILE diagnostic under static or radiofrequency electric field in vacuum. Plasma Science and Technology, 2018, 20 (7). hal-01783151

\section{HAL Id: hal-01783151 \\ https://hal.science/hal-01783151}

Submitted on 2 May 2018

HAL is a multi-disciplinary open access archive for the deposit and dissemination of scientific research documents, whether they are published or not. The documents may come from teaching and research institutions in France or abroad, or from public or private research centers.
L'archive ouverte pluridisciplinaire HAL, est destinée au dépôt et à la diffusion de documents scientifiques de niveau recherche, publiés ou non, émanant des établissements d'enseignement et de recherche français ou étrangers, des laboratoires publics ou privés. 


\title{
Estimation of the Lyman- $\alpha$ signal of the EFILE diagnostic under static or radiofrequency electric field in vacuum
}

\author{
Carlo POGGI, ${ }^{1}$ Théo GUILlaUME, ${ }^{2}$ Fabrice \\ DOVEIL, ${ }^{2}$ and Laurence CHÉRIGIER-KOVACIC ${ }^{* 2}$ \\ ${ }^{1}$ Consorzio RFX, Corso Stati Uniti, 35127 Padova, Italy \\ ${ }^{2}$ Aix-Marseille Université, CNRS, PIIM UMR 7345, \\ FR-13397 Marseille Cedex 20, France
}

(Dated: April 27, 2018)

\begin{abstract}
The EFILE diagnostic (Electric Field Induced Lyman- $\alpha$ Emission) aims to provide a non intrusive and precise measurement of the electric field in plasma, using a beam of hydrogen atoms prepared in the metastable $2 s$ state. The metastable particles are obtained by means of a proton beam extracted from a hydrogen plasma source, and neutralised by interaction with vaporised caesium. When a $2 s$ atom enters a region where an electric field is present, it undergoes a transition to the $2 p$ state (Stark mixing). It then quickly decays to the ground level, emitting Lyman- $\alpha$ radiation, which is collected by a photomultiplier. The $2 s \rightarrow 2 p$ transition rate is proportional to the square of the magnitude of the electric field, and depends on the field oscillation frequency (with peaks around $1 \mathrm{GHz}$ ). By measuring the intensity of the Lyman- $\alpha$ radiation emitted by the beam it is possible to determine the magnitude of the field in a defined region.

In this work, an analysis of the behaviour of the diagnostic under static or radiofrequency electric field is presented. Electric field simulations obtained with a finite element solver of Maxwell equations, combined with theoretical calculations of the Stark mixing transition rate, are used to develop a model for the interpretation of photomultiplier data. This method shows good agreement with experimental results for the static field case, and allows to measure the field magnitude for the oscillating case.
\end{abstract}

Keywords: Stark effect, Electric field measurement, Diagnostics, Electromagnetic simulations

\footnotetext{
* Corresponding author. Email address: laurence.kovacic@univ-amu.fr
} 


\section{Introduction}

The EFILE (Electric Field Induced Lyman- $\alpha$ Emission) diagnostic is currently under development at PIIM laboratory at Aix-Marseille Université (France). It aims to provide a direct, non intrusive and precise measurement of a static or oscillating electric field in vacuum and in a plasma, using a beam of hydrogen atoms prepared in the metastable $2 s$ state. It is of considerable importance to determine the electric field involved for example in sheaths or plasma-wall interactions[1], plasma thrusters[2], plasma turbulence, but such diagnostics are rare. A detailed set-up description of the EFILE diagnostic can be found in [3]. We have already measured the absolute value of the static electric field profile between two polarized plane electrodes in vacuum with a good agreement with a numerical calculation of the field. We have applied the same technique to measure the static electric field between two polarized plane electrodes in a plasma, where the field is null (quasineutrality) except in the sheath near the polarized electrode [4. The sheath thickness has been related to the plasma density. Now we are adressing the problem of oscillating fields. Using one of the electrode as an emitting antenna by applying an oscillating potential with a frequency varying in the range 800-1600 $\mathrm{MHz}$, we observe very narrow peaks (width less than 40 $\mathrm{MHz},[5]$ ) at frequencies depending on the presence or not of metallic components inserted in the chamber. We conclude that these peaks could be resonance modes of the chamber, actually being a cavity of about $30 \mathrm{~cm}$ with a complex geometry. In this article we present a simulation of our experimental device including the measurement process and we compare the numerical results to the experimental ones. We confirm in particular that we measure the RF electric field associated to resonance modes of the cavity and draw conclusions about a possible way to calibrate the measurements.

Let us first briefly recall the principle of this diagnostic. Metastable particles are obtained by means of a proton beam extracted from a hydrogen plasma source, and neutralised by interaction with vaporised cesium. When a $2 s$ atom enters a region where an electric field is present, it undergoes a transition to the $2 p$ state (Stark mixing). Then it quickly decays to the ground level, emitting Lyman- $\alpha$ radiation, which is collected by a VUVphotomultiplier. The $2 s \rightarrow 2 p$ transition rate is proportional to the square of the magnitude of the electric field, and depends on the field oscillation frequency (with peaks around $1 \mathrm{GHz}$ ). 
Its expression was estimated in [3] and refined in [6]

$$
\gamma\left(E_{0}, \omega\right)=3\left(\frac{a_{0} e E_{0}}{\hbar}\right)^{2} \frac{\Gamma}{\left(\frac{\Gamma}{2}\right)^{2}+\left(\omega-\omega_{L}\right)^{2}}
$$

where $a_{0}$ is the Bohr radius, $e$ is the elementary charge, $\hbar$ is the reduced Plank constant, $\Gamma=$ $100 \mathrm{MHz}$ is the transition rate of the $2 p \rightarrow 1 s$ process and $\omega_{L} / 2 \pi=1.058 \mathrm{GHz}$ corresponds to the energy difference between the $2 s$ and $2 p$ states (Lamb shift [7], 8], 9]); $E_{0}$ and $\omega$ are respectively the amplitude (which can eventually vary in space, $E_{0}(\vec{r})$ ) and the angular velocity of an electric field expressed as $E=E_{0} \cos (\omega t)$. This formula is valid in a small field approximation [10], i.e. for $E_{0} \ll 26 \mathrm{kVm}^{-1}$ when $\omega=0$ (static field) and $E_{0} \ll 1.3 \mathrm{kV} \mathrm{m}^{-1}$ when $\omega=\omega_{L}$, and the quadratic dependence on the electric field amplitude was confirmed in previous works [1].

The intensity of the Lyman- $\alpha$ radiation emitted by the beam depends on this transition rate. By measuring the light intensity it is therefore possible to determine the magnitude of the field in the region defined by the crossing of the collecting photomultiplier view with the beam.

\section{Analytical model}

Starting from the transition rate of equation (1), the number of decaying atoms per unit of time in the test volume (viewed by the photomultiplier) was calculated to estimate the radiation intensity [3]. We supposed a constant field along the path of the beam, assimilated to a line running in the middle between the $5 \mathrm{~cm}$-spaced electrodes. We now refine this model in order to take into account the spatial extension of the beam and distribution of the electric field [6]. Let the beam be generated at $z=z_{s}$ and described by a $\varphi_{2 s}(x, y, z)$ flux of metastable particles, moving along the $z$ axis with non relativistic speed $\vec{v}=(0,0, v)$. The variation of $\varphi_{2 s}$ is given by

$$
\mathrm{d} \varphi_{2 s}(\vec{r})=-\gamma(\vec{r}, \omega) \varphi_{2 s}(\vec{r}) \mathrm{d} t=-\gamma(\vec{r}, \omega) \varphi_{2 s}(\vec{r}) \frac{\mathrm{d} z}{v}
$$

assuming it is only due to the Stark-mixing effect. The integration gives

$$
\varphi_{2 s}(\vec{r})=\varphi_{2 s, z_{s}}(x, y) \exp \left(-\int_{z_{s}}^{z} \gamma \frac{\mathrm{d} z^{\prime}}{v}\right)
$$

where $\varphi_{2 s, z_{s}}(x, y)$ is the flux of particles at $z=z_{s}$. The flow rate is then obtained integrating 
over the beam section $\Sigma$

$$
N_{2 s}(z)=\int_{\Sigma} \varphi_{2 s}(\vec{r}) \mathrm{d} x \mathrm{~d} y=\int_{\Sigma} \varphi_{2 s, z_{s}}(x, y) \exp \left(-\int_{z_{s}}^{z} \gamma \frac{\mathrm{d} z^{\prime}}{v}\right) \mathrm{d} x \mathrm{~d} y
$$

Assuming that the photomultiplier can view the region of the beam from $z_{0}$ to $z_{0}+\Delta z$, the intensity $I$ of the radiation emitted by the beam and detected is proportional to the opposite of the variation of $N_{2 s}(z)$ in this interval:

$$
I=-\beta \Delta N_{2 s}=\beta \int_{\Sigma} \varphi_{2 s, z_{s}}(x, y) e^{-\int_{z_{s}}^{z_{0}} \gamma \frac{\mathrm{d} z}{v}}\left(1-e^{-\int_{z_{0}}^{z_{0}+\Delta z} \gamma \frac{\mathrm{d} z}{v}}\right) \mathrm{d} x \mathrm{~d} y
$$

and the proportionality coefficient $\beta$ is related to the photomultiplier properties (mainly efficiency and solid angle). This equation holds for a completely general electric field configuration. To make a measurement in the $z_{0}<z<z_{1}$ region, we must take into account the decay of the atoms in the probe beam before $z_{0}$. Indeed, the signal intensity, given by the number of emitting atoms detected in the diagnosed volume, depends on the history of the beam, since atoms will decay all along their path. Given the coefficient $\beta$ and the density distribution $\varphi_{2 s}\left(x, y, z_{s}\right)$ of the beam, a relation between the electric field in the measurement region and the Lyman- $\alpha$ signal can be established given the spatial field distribution upstream.

\section{Simulations and comparison with experimental data}

As shown in the previous section, the Lyman- $\alpha$ signal is strongly dependent on the spatial distribution of the electric field. The CST Studio Suite software is used to create a 3D model of the test chamber and to solve Maxwell equations in vacuum, when a static or RF voltage is applied between the electrodes. The magnitude of the field needed to estimate the light emission detected with the photomultiplier is computed. Figure 1 shows the geometry of the chamber as well as the field map for different frequencies. The vessel is cylindrical with a smaller cylinder attached at the bottom of the main vessel, leading to the vacuum pump (not represented on the figure). The plates are held by a grounded vertical rod and centered about two horizontal tubes on each side of the vessel. The lower electrode is the small rectangle which seems to be floating, but it is actually linked to the upper electrode by an insulated rod which is not shown on the figure because it is behind the plane of the displayed results. In the real experimental device, the atomic beam travels in the left tube and passes between the electrodes. A small probe can be inserted in the chamber from 
the right horizontal tube in order to perform electrical measurement of the electric field spectrum. Its presence (or absence) in the vessel also modifies the geometry and thus the resonant frequencies of the vessel [6].

We represent in figure 1 the electric field magnitude in vacuum for three different frequencies (static case, $1.252 \mathrm{GHz}$ and $1.400 \mathrm{GHz}$ ). As expected, it depends on the position in the vessel and on the vessel eigenmodes which are excited.

\subsection{Static field}

Static field measurements were made applying a voltage $V$ to the lower plate in the range from $-500 \mathrm{~V}$ to $500 \mathrm{~V}$ (such that the maximum simulated value of the electric field along the beam line was $13 \mathrm{kV} \mathrm{m}^{-1}$ ). The results were interpolated using equation (5) where the integral is calculated using $\gamma(\vec{E})$ with the electric field obtained from the simulations and including a shift corresponding to the signal at $V=0$. This signal is due to the spontaneous emission of the beam in the Lyman- $\alpha$ wavelength range.

The fitting parameters are $\beta, C$ (the shift), $z_{0}$ and $\Delta z$, while $z_{s}$ is fixed (it was chosen such that $\left.E\left(x, y, z_{s}\right) \approx 0\right)$. The beam profile $n_{2 s}\left(x, y, z_{s}\right)$ is a circle of $r=10 \mathrm{~mm}$ radius [3], and its value is set at $1 /\left(\pi r^{2}\right)$ inside the circle and 0 outside. The integration area $\Sigma$ is defined by the active surface of the photomultiplier: it is a rectangle of $8 \mathrm{~mm}$ height and $12 \mathrm{~mm}$ width. The integration along the vertical axis has been fixed to a $8 \mathrm{~mm}$ gap (from $-4 \mathrm{~mm}$ to $4 \mathrm{~mm})$.

In figure 2 two measurements made at different dates are presented, together with the interpolating curves. The signal shape is the same for both datasets. The signal at $V=0$ depends on the beam characteristics which were different in the two measurements, as well as the parameter $\beta$. The fit results are

$$
\begin{array}{r}
z_{0,1}=(-9.0 \pm 0.1) \mathrm{mm} \\
(\Delta z)_{1}=(10.78 \pm 0.07) \mathrm{mm} \\
\beta_{1}=(9.99 \pm 0.06) \mathrm{mV} \\
C_{1}=(0.097 \pm 0.002) \mathrm{mV}
\end{array}
$$




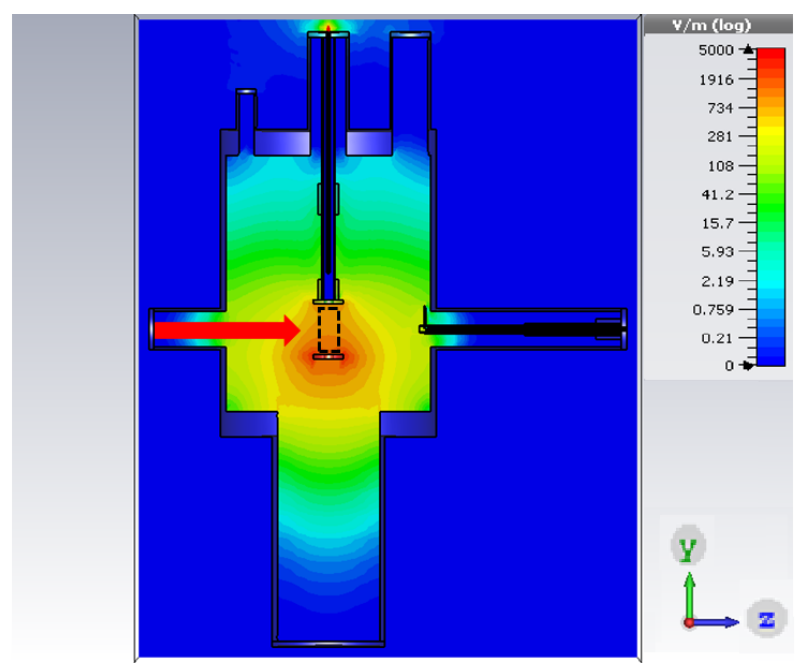

(a) Static case

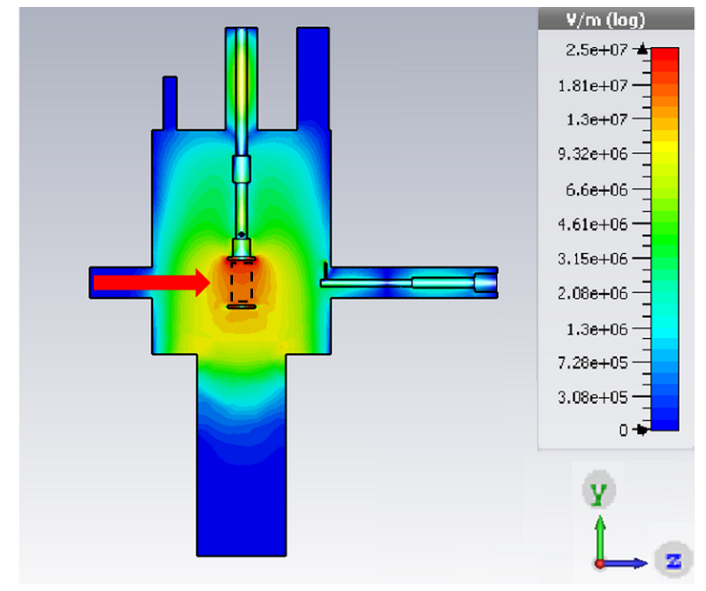

(b) $1.252 \mathrm{GHz}$

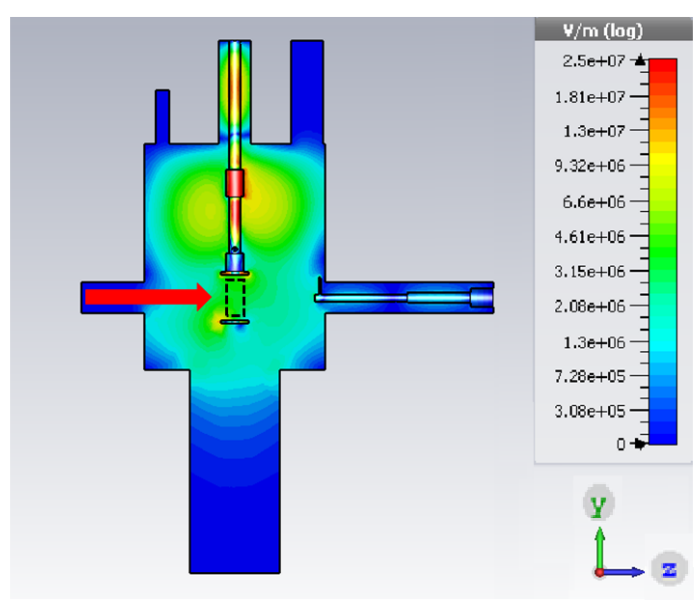

(c) $1.400 \mathrm{GHz}$

Figure 1: Projections of the amplitude (in logarithmic scale) of the electric field on the plane perpendicular to the beam for the static case (with $50 \mathrm{~V}$ applied to the lower plate) and for two eigenvalues. For the resonant cases, the amplitude of the field is such that the entire energy stored in the electromagnetic field inside the vessel is $1 \mathrm{~J}$. The red arrow in each picture represents the beam line, and the black rectangle shows the region detected by the photomultiplier. 


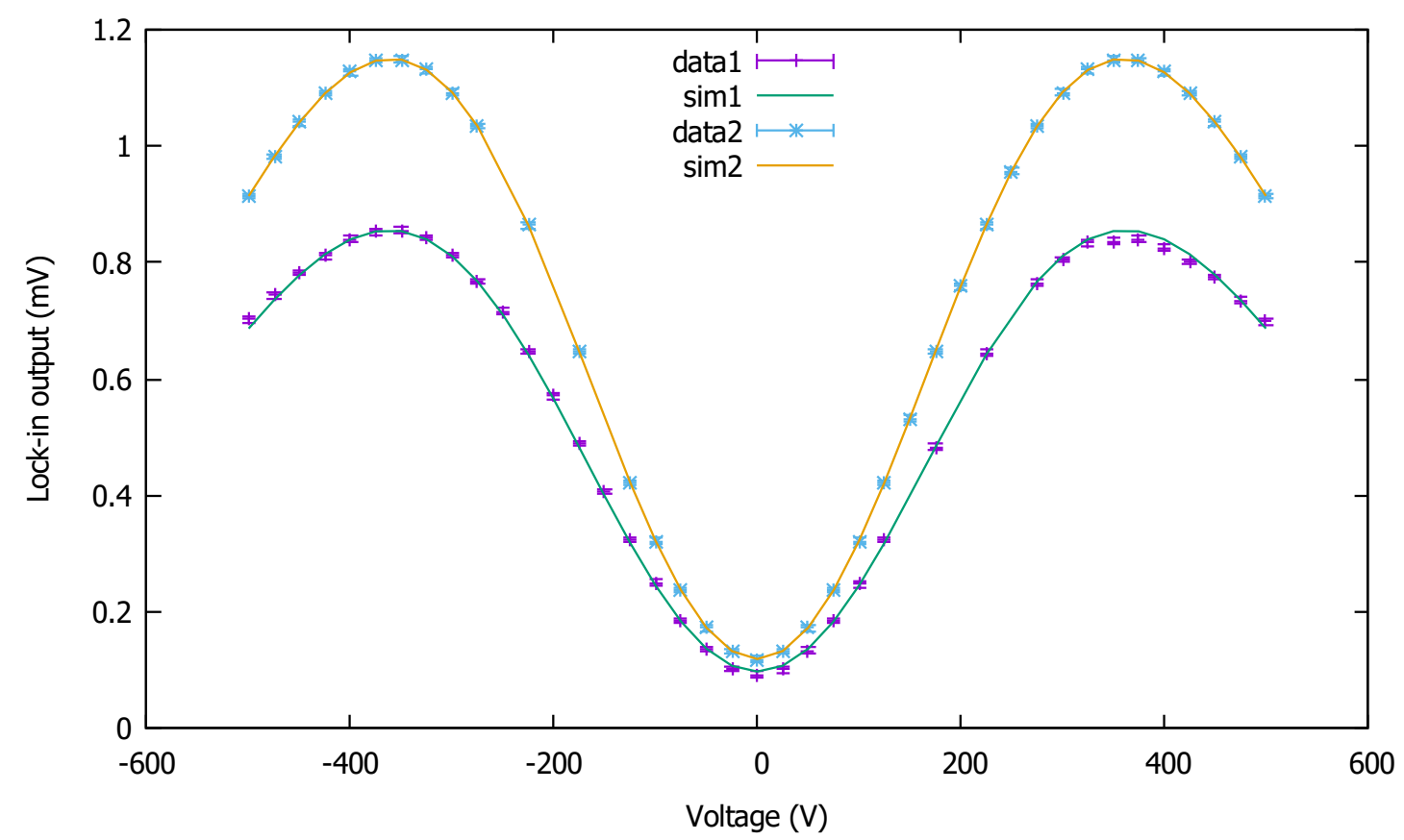

Figure 2: Comparison with the simulated output.

for the first set and

$$
\begin{aligned}
z_{0,2} & =(-9.95 \pm 0.09) \mathrm{mm} \\
(\Delta z)_{2} & =(13.53 \pm 0.09) \mathrm{mm} \\
\beta_{2} & =(10.96 \pm 0.06) \mathrm{mV} \\
C_{2} & =(0.118 \pm 0.002) \mathrm{mV}
\end{aligned}
$$

for the second one.

As it can be seen, the experimental signal presents a saturation (at a voltage of $360 \mathrm{~V}$ ), due to the decay of the beam by the field before the detection volume. The simulated curves are adherent to the respective datasets, and the $\Delta z$ are comparable with the expected width of the photomultiplier $(12 \mathrm{~mm})$, while the integration range along the $z$ axis is shifted and it is not symmetric with respect to 0 . The reason might be a misalignment of the photomultiplier with respect to the plates center (it would be tilted of a few degrees), or it might be due to the different radial profile of the beam, that for fitting necessities was simply considered as a homogeneous circle of fixed diameter. 


\subsection{RF field}

RF tests were performed after the static field measurements. The lower plate was polarised with a radiofrequency signal with a frequency between 0.8 and $1.6 \mathrm{GHz}$, with a maximum constant power of $P_{\max }=33 \mathrm{dBm}=2 \mathrm{~W}$ delivered to the electrode. If the magnitude of the electric field in the vessel would have been independent of its frequency, we would have retrieved the Lorentzian curve of eq. (1). In figure 3 , the experimental spectrum is reported. As it can be seen, the frequency behaviour is different from the expected Lorentzian. We observe a series of very thin peaks with a baseline corresponding, like in the static case, to the spontaneous emission of the atomic probe beam. This signal level is the same in the absence of RF power and we conclude that the electric field is null between the peaks.

The fitting parameters of the previous section depend on the properties of the beam, and were determined from the static field measurements. This fitting procedure was possible because the voltage of the lower plate was known, and therefore the intensity of the simulated electric field was realistic. Instead, the RF polarisation of the lower plate strongly depends on the transmission line connecting the RF generator to the lower plate, which could not be properly simulated since losses were not taken into account in the simulation. Therefore the field intensity simulated for each frequency was not reliable, and a fitting procedure for the RF case could not be performed. A numerical estimation of the RF signal was obtained integrating eq. (5) with the parameters taken from the static fit, and the electric field simulated for each frequency, assuming the spatial distribution of the field, which depends on the excited eigenmode, was correctly reproduced by the simulation. The simulated spectrum corresponding to the static dataset 2 described before is superposed in figure 3. Peaks corresponding to resonant frequencies in the cavity are observed.

The shape of the peaks is not exactly reproduced: the simulated peaks are higher and thinner than the experimental data. This is due to the presence of losses in the transmission line, which broaden and lower the resonances and are not considered in the simulation.

Moreover, the experimental peaks are not placed at the same frequencies as the simulated ones, except for the peaks at $1.026 \mathrm{GHz}, 1.252 \mathrm{GHz}$ and at $1.400 \mathrm{GHz}$. This is due to the sensitivity of the resonant frequencies to geometrical details [6]. However, for the three experimental peaks mentioned before a correspondence with the simulation was found and, 


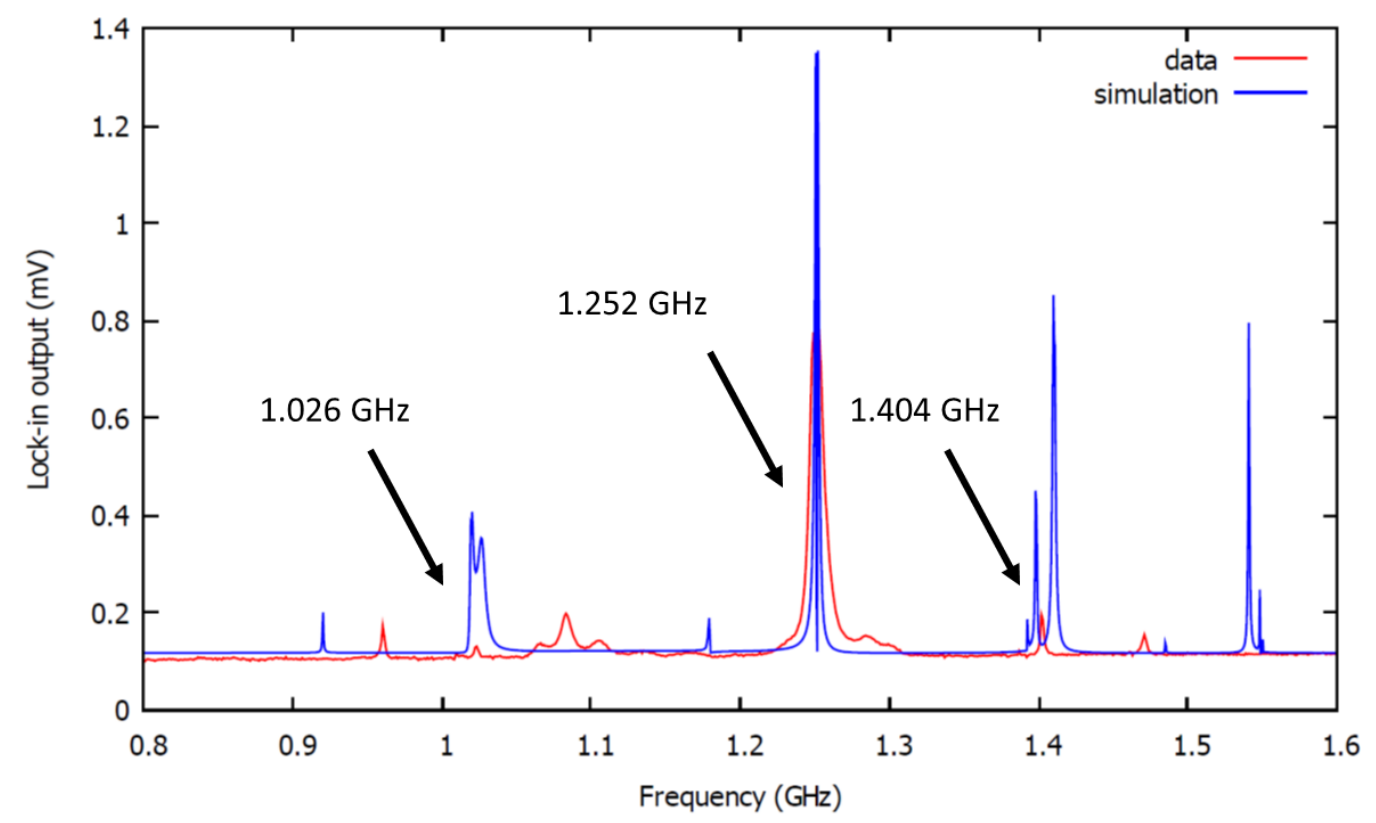

Figure 3: Comparison between the experimental and the simulated Lyman- $\alpha$ spectra. The peaks for which a correspondence was found are indicated.

although the amplitude of the field could not be estimated properly, since losses were not taken into account, we could obtain the spatial distribution of the excited eigenmode from the simulation.

Several measurements for different injected powers were performed to study the behaviour of the emitted radiation at the frequencies of the three peaks. The measurements for the $1.026 \mathrm{GHz}$ resonance could hardly be distinguished from the background, and therefore are not considered. The other two datasets are presented in figure 4 (a) and (b).

To compare the experimental data with the simulations, the field maps of the electric field of the peaks obtained from an eigenvalues simulation were used: the simulated electric field $E_{\text {eigen }}$ was multiplied by different factors $m$ and then equation 5 was integrated for every $m$, using the parameters determined for the static case fit. This way the expected behaviour of the signal was reproduced. Then the relation

$$
P=\alpha E^{2}
$$

between power and electric field was used to superpose data and simulations, exploiting the property of logarithmic units such as $\mathrm{dBm}$. Therefore the abscissa of the experimental and simulated data only differ by a constant, which was determined by imposing the coincidence 


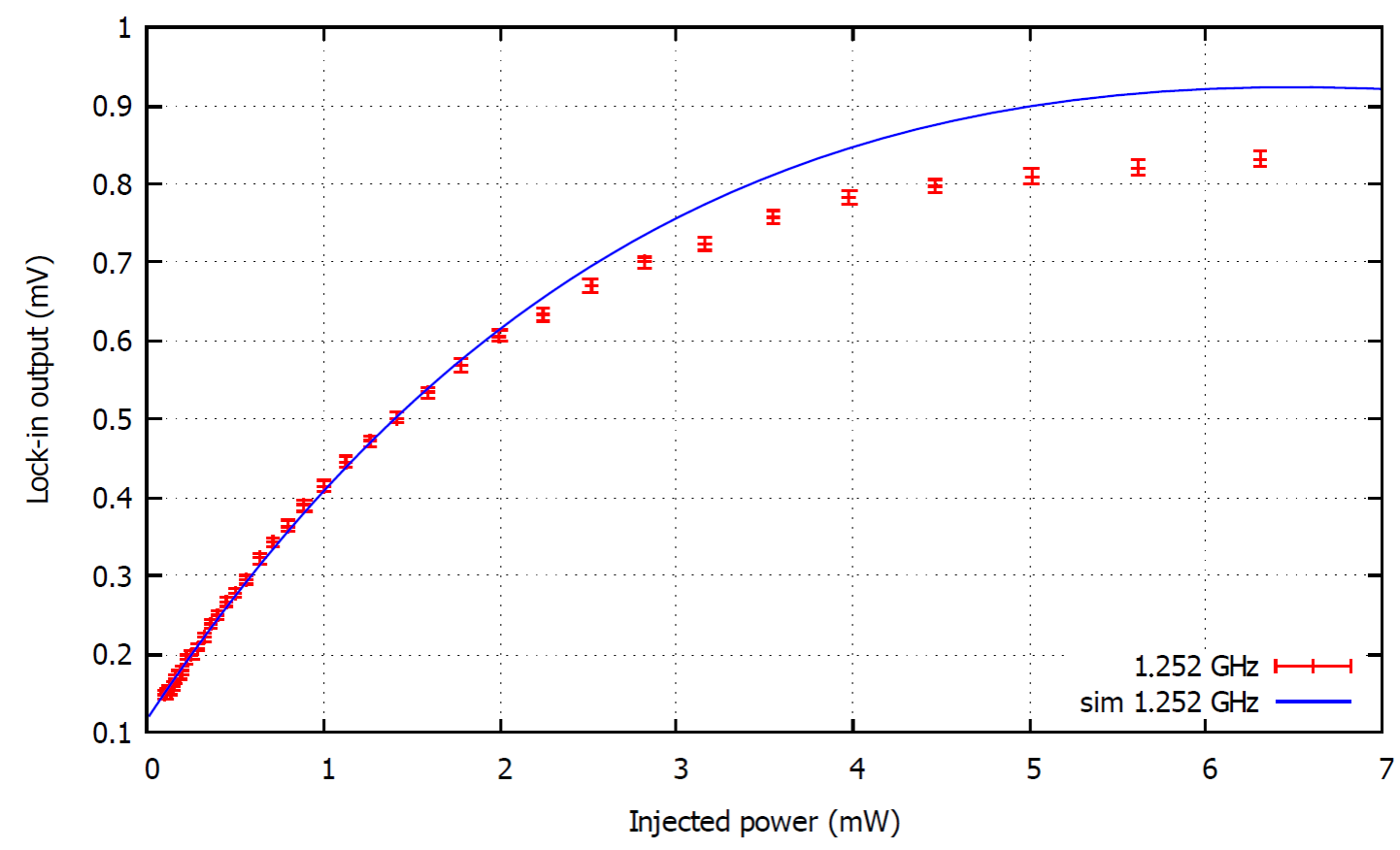

(a) $1.252 \mathrm{GHz}$ peak.

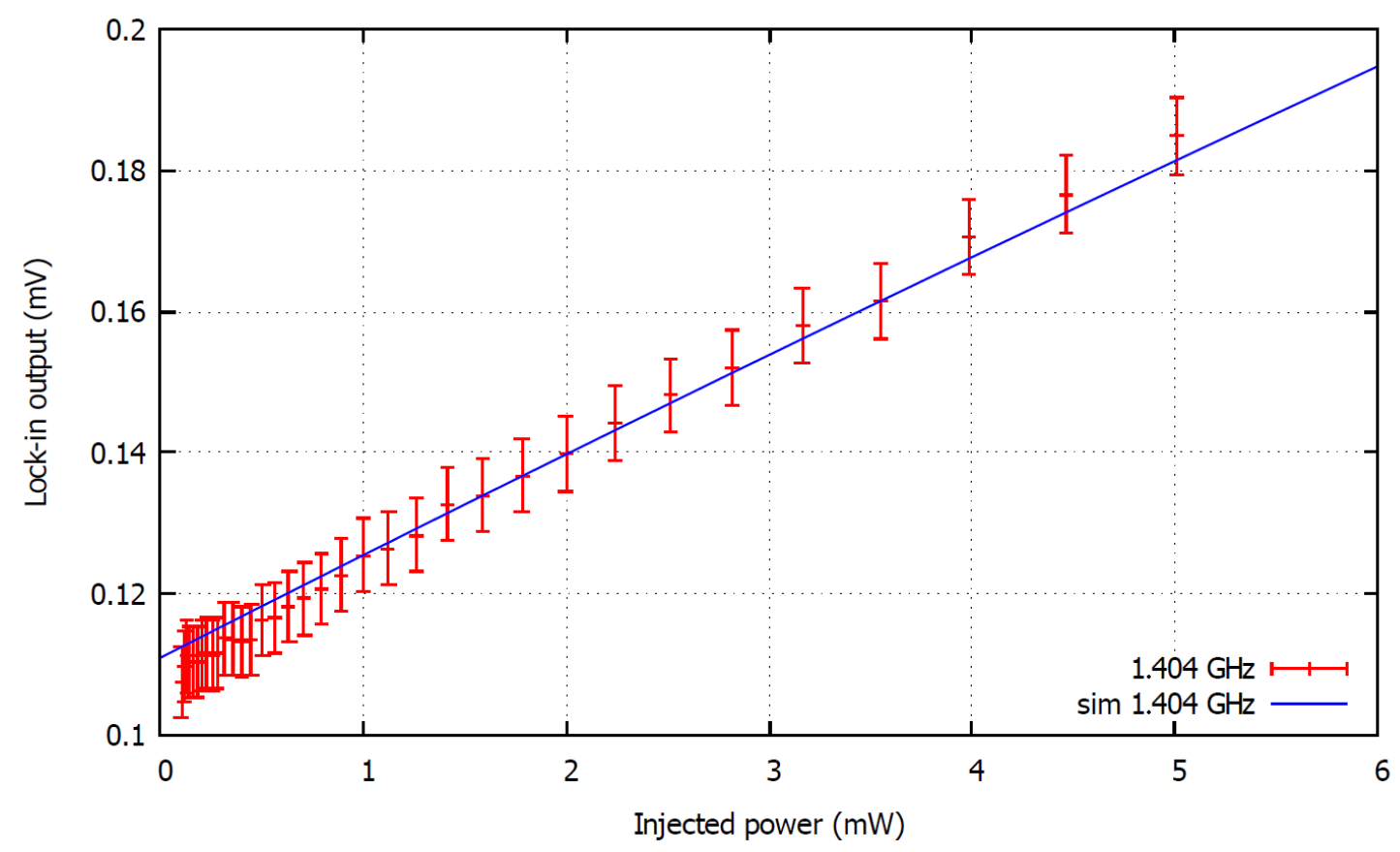

(b) $1.404 \mathrm{GHz}$ peak.

Figure 4: Lyman- $\alpha$ measurements for different injected powers at fixed frequencies and comparison with the simulations. Both experimental and simulated data are linear for small values of the injected power, as expected since both the transition rate and the injected power are proportional to $E^{2}$. 
of one simulated and one experimental value.

As it can be seen, the data are well described by the simulation in the region before the saturation. Only the $1.252 \mathrm{GHz}$ peak approaches the saturation, but at those values it moves away from the simulation. This is probably due to a variation of beam properties with time, as these measurements were performed later than the one with static field. The values were then normalized by subtracting the spontaneous emission constant and dividing by the highest value recorded for the $1.252 \mathrm{GHz}$ case, and the results, reported in figure 4 (c) and (d), show a good agreement between simulation and experiment. Therefore, a correspondence between the simulated electric field and the Lyman- $\alpha$ radiation detected was found.

\section{Conclusion}

This work focuses on the use of a numerical simulation to understand experimental results. The experimental device was simulated and the frequencies of the resonance modes as well as the map of the static and RF (up to $1.6 \mathrm{GHz}$ ) electric field generated in the vessel were computed. An analytical model of the measurement process was developed, and the Lyman$\alpha$ intensity as a function of the frequency of the field was estimated.

Measurements and computed signal fit together very well in the static field case, assessing the correctness of the model and of the electric field simulation given the theoretical transition rate of the metastable level, and demonstrating that the knowledge of the topology of the field is required to correctly reproduce the experimental behaviour. If the spatial distribution is not known, a solution could be to sample the Lyman-alpha signal at multiple positions along the beam. This is presently not possible in our test device but will be studied in the frame of a future project. For the RF case, we gave a qualitative explanation of the observed spectrum in terms of resonant frequencies of the experimental chamber, and we found a correspondence between the simulated electric field and the Lyman- $\alpha$ signal as a function of the injected power for two eigenfrequencies with different field topologies.

However, the agreement of the model with the data suggests that this diagnostic is adapted to measure static or RF electric fields, once a trusty calibration method will be found, which is part of the work in progress. It could be used, for example, to measure the electric field amplitude generated in front of an ICRF heating antenna, for which the field 
topology is given by electromagnetic simulations.

\section{Acknowledgments}

We thank Guillaume Demesy, Pierre Sabouroux and Marc Dubois of the Fresnel Institute for fruitful discussions and for technical support with CST. C. P. benefited from an Erasmus exchange grant via the European Erasmus+ Programme 2014-2020.

[1] Samarian A A et al. 2005, Plasma Phys. Control. Fusion 47 B629

[2] Dudeck M et al. 2012, IOP Conf. Ser.: Mater. Sci. Eng. 29012010

[3] Chérigier-Kovacic L et al. 2015, Review of Scientific Instruments 86, 063504

[4] Doveil F et al. 2017, Plasma physics and Controlled Fusion 59, 014020

[5] Doveil F, et al. 2013, Physics of Plasmas 20055701

[6] Poggi C 2017, M.S. thesis, Università degli Studi di Padova

[7] Lamb W E Jr and Retherford R C 1947, Phys. Rev. 72241

[8] Lamb W E Jr and Retherford R C 1950, Phys. Rev. 79549

[9] Lamb W E Jr. 1951, Rep. Prog. Phys. 1419

[10] Ström P 2013, M.S. thesis, Aix-Marseille Université

[11] Lejeune A et al. 2011, Applied Physics Letters 99181502 